\title{
Aplicação da Metodologia para Qualificação de Procedimentos de Soldagem de Tubulações Industriais Conforme Parâmetros dos Códigos ASME B31.3 e ASME SECTION IX*
}

\author{
Ana Carla S. Silva ${ }^{1}$ (D), Helen R. Araújo ${ }^{1}$ (D), Vagner A. Macena², João R. Sartori Moreno ${ }^{3}$ (D), André A. Vicente ${ }^{4}$ (D), \\ Tahiana F. C. Hermenegildo 5 (D), Tiago F. A. Santos ${ }^{1}$ (D) \\ ${ }^{1}$ Universidade Federal de Pernambuco - UFPE, Departamento de Engenharia Mecânica, Recife, PE, Brasil. \\ 2 Serviço Nacional de Aprendizado Industrial - SENAI, Caruaru, PE, Brasil. \\ ${ }^{3}$ Universidade Tecnológica Federal do Paraná - UTFPR, Departamento de Engenharia Mecânica, Cornélio Procópio, PR, Brasil. \\ ${ }^{4}$ Escola Politécnica da Universidade de São Paulo - EPU, Departamento de Engenharia Química, São Paulo, SP, Brasil. \\ ${ }^{5}$ Universidade Federal do Rio Grande do Norte - UFRN, Departamento de Engenharia Mecânica, Natal, RN, Brasil.
}

Como citar: Silva ACS, Araújo HR, Macena VA, Moreno JRS, Vicente AA, Hermenegildo TFC, Santos TFA. Aplicação da metodologia para qualificação de procedimentos de soldagem de tubulações industriais conforme parâmetros dos códigos ASME B31.3 e ASME SECTION IX. Soldagem \& Inspeção. 2019;24:e2423. https://doi.org/10.1590/0104-9224/SI24.23

\begin{abstract}
Resumo: Este trabalho foi desenvolvido visando auxiliar os profissionais de soldagem quanto as incertezas relacionadas às atividades de fabricação, montagem e manutenção de tubulações em plantas industriais no qual se refere à maneira adequada para a condução de atividades de qualificação de procedimentos de soldagem. Discorre sobre a metodologia de qualificação de procedimentos de soldagem de tubulações industriais conforme os parâmetros dos códigos ASME B31.3 (Tubulação de Processos), sobre trabalhos executados em tubulações de processos industriais e ASME BPV Code SECTION IX (Qualificação de Soldagem, Brasagem e Fusão), norma largamente empregada para a qualificação de operadores e procedimentos de soldagem. O enfoque desse estudo é uma abordagem interpretativa destas normas visando à aplicabilidade para a qualificação de procedimento de soldagem destinados à fabricação de tubulações industriais. A condução desta qualificação, em conformidade com as normas citadas, apresentou resultados bastante satisfatórios dos corpos-de-prova submetidos aos ensaios mecânicos de dobramentos e de resistência à tração. Esses resultados demonstraram a assertividade das variáveis de soldagem empregadas na qualificação a partir dos códigos utilizados. Viabiliza-se, ainda, possibilidades do emprego desse material como complemento e apoio didático nos currículos de engenharia - mais precisamente - nas disciplinas de processos de fabricação.
\end{abstract}

Palavras-chave: Qualificação de procedimento de soldagem; ASME B 31.3; ASME SECTION IX; Tubulações industriais.

\section{Applied Methodology for Qualification of Welding Procedures of Industrial Pipes According to ASME B 31.3 and ASME SECTION IX}

\begin{abstract}
This work was developed to help welding professionals concerning the uncertainties related to the activities of manufacturing, assembly and maintenance of pipes in industrial plants in which it refers to the proper way to conduct activities to qualify welding procedures. It discusses about the methodology for the qualification of industrial tube welding procedures according to the parameters of the ASME B31.3 code (Processes Piping), about work performed on industrial process pipes and ASME BPV code SECTION IX (Welding, Brazing and Fusing Qualification), widely used for the qualification of welders and welding procedures. The purpose of this study is an interpretative approach of these codes aiming at the applicability of the qualification of welding procedure intended for the manufacture of industrial pipes. The conduction of this qualification, in accordance with the cited codes, presented quite satisfactory results of the specimens submitted to the mechanical bend and tensile strength tests. These results demonstrated the confidence of the welding variables used in the qualification from the codes used. It also makes possible the use of this material as a complement and didactic support in engineering curriculum - more precisely - in the disciplines of manufacturing processes.
\end{abstract}

Key-words: Qualification of welding procedure; ASME B 31.3; ASME SECTION IX; Industrial pipes.

Recebido: 20 Dez., 2018. Aceito: 23 Ago., 2019

E-mail: carla.santoss@ufpe.br (ACSS)

*Contribuição para XLIV CONSOLDA - Congresso Nacional de Soldagem sob título: “Metodologia para Qualificação de Procedimentos de Soldagem de Tubulações Industriais Conforme Parâmetros dos Códigos ASME B31.3 e ASME SECTION IX"

(i) (\$) Este é um artigo publicado em acesso aberto (Open Access) sob a licença Creative Commons Attribution Non-Commercial, que permite uso, distribuição e reprodução em qualquer meio, sem restrições desde que sem fins comerciais e que o trabalho original seja corretamente citado. 


\section{Introdução}

Destacadamente, a soldagem é um dos métodos de união de metais mais empregados industrialmente [1]. Por sua vez, a mesma é caracterizada como um processo de fabricação de larga amplitude de aplicação, logo não é possível aos engenheiros e soldadores uma previsão absoluta do resultado desta operação, pois, sabe-se que, mesmo com o emprego de altas tecnologias, a presença de descontinuidades ou defeitos é uma característica recorrente neste tipo de união.

A ocorrência de problemas na soldagem devido ao emprego de procedimentos inadequados pode ocasionar defeitos nos cordões de solda (por exemplo: trincas, faltas de fusão e inclusões), impactar negativamente na vida útil e funcionamento de equipamentos industriais, provocar acidentes com impactos ao ambiente e até causar a mortes, bem como paradas nãoprogramadas do setor produtivo[1].

Portanto, faz-se necessário a devida qualificação dos procedimentos em conformidade com códigos e normas técnicas regulamentadoras dos mesmos envolvidos nas operações de soldagem. Tal detalhamento engloba desde a preparação da junta, na execução da soldagem e segue até à realização de testes e ensaios necessários à verificação da junta soldada. As informações contidas no detalhamento do processo de soldagem devem ser registradas a fim de obter o máximo de repetibilidade das propriedades mecânicas da união soldada, quando executada por profissionais devidamente treinados [2, 3]. A norma ASME IX define quais são os documentos que são utilizados para o registro adequado do procedimento de soldagem [4].

Dentro deste contexto, neste trabalho foi realizado um estudo prático baseado nos códigos ASME B3.1 e ASME SECTION IX visando à aplicabilidade destes elementos normativos no direcionamento adequado para a qualificação de procedimentos de soldagem destinados à fabricação de tubulações industriais.

\section{Documentação de Soldagem}

A coleta dos dados para o desenvolvimento dos corpos de prova teste e dos documentos de soldagem: Registo de Qualificação de Procedimento de Soldagem (RQPS) e Especificação de Procedimento de Soldagem (EPS), utilizados como estudo de caso neste trabalho, foi orientada por meio dos códigos ASME B31.3 e ASME SECTION IX [4, 5]. O código ASME IX define as variáveis do processo de soldagem em três tipos, conforme Tabela 1.

Tabela 1. Definição do tipo de variável de processo de soldagem conforme ASME IX

\begin{tabular}{cl}
\hline Tipo de Variável & \multicolumn{1}{c}{ Característica } \\
Essenciais & $\begin{array}{l}\text { São aquelas cuja variação, além dos limites permissíveis, poderão afetar as } \\
\text { propriedades mecânicas da junta soldada e exige nova qualificação da EPS. } \\
\text { São aquelas cuja alteração nas condições de soldagem poderão afetar as }\end{array}$ \\
propriedades da resistência ao impacto da junta soldada e a mudança destas \\
variáveis, além do limite permissível e quando aplicável, também exige nova \\
qualificação da EPS.
\end{tabular}

Os principais documentos para a qualificação do procedimento de soldagem são o RQPS e a EPS. Estes documentos são previstos nas normas técnicas cuja finalidade é reunir informações necessárias para execução do processo de soldagem para aplicações em tubulações e equipamentos industriais, sempre em conformidade com os códigos de projeto, fabricação ou inspeção [4].

No Brasil, em se tratando de fabricação de tubulações industriais, especialmente tubulações de processo empregadas em, por exemplo, refinarias de petróleo e petroquímicas, os profissionais habilitados para elaboração e validação do RQPS e da EPS são os Inspetores de Soldagem Nível 2 ou os Engenheiros de Soldagem Especialistas. Estes profissionais devem estar devidamente qualificados e certificados, conforme NBR 14842 pelo organismo de certificação de pessoal (OCP), onde no Brasil é realizado pela Fundação Brasileira de Tecnologia da Soldagem (FBTS), órgão este acreditado pelo INMETRO [6]. Os tópicos seguintes discorrem sobre a correta interpretação destes códigos normativos visando a qualificação de procedimentos de soldagem para aplicação, mais especificamente, em tubulações industriais.

\subsection{Registo de Qualificação de Procedimento de Soldagem (RQPS)}

O RQPS é o registro dos dados utilizados para soldar uma peça de teste (que poderá ser chapa ou tubo). Neste documento, devem ser registrados os valores reais das variáveis essenciais de soldagem e, quando requerido, das variáveis suplementares e não essenciais efetivamente empregadas na soldagem da peça de teste. Deve conter, ainda, todos os resultados dos ensaios e testes mecânicos realizados como requisito para qualificação do procedimento de soldagem. O RQPS somente é considerado 
válido depois que os resultados dos testes e ensaios exigidos forem considerados aprovados de acordo com valores especificados por norma. O modelo de formulário está disponível no QW-483 do código ASME SECTION IX [4].

\subsection{Especificação de Procedimento de Soldagem (EPS)}

A EPS é um documento que registra o procedimento de soldagem escrito e qualificado. Este documento é emitido a partir de uma RQPS. Destina-se ao fornecimento das informações quanto à técnica de soldagem a ser utilizada para a execução de soldas em atendimento aos requisitos das normas de fabricação e das normas de qualificação de procedimento de soldagem. O modelo de formulário de EPS está disponível no QW-482 [4].

\section{Normas Utilizadas}

No Brasil, o órgão que regulamenta os procedimentos, os processos e os ensaios é a Associação Brasileira de Normas Técnicas (ABNT). Entretanto, as normas internacionais ASME são muito empregadas na fabricação de tubulações de processos em refinarias e indústrias petroquímicas, dentre as quais se destacam:

- $\quad$ O código ASME B31.3: Tubulação de Processos - Utilizado para direcionar os trabalhos de tubulações de processos industriais [5];

- O código ASME SECTION IX: Qualificação de Soldagem, Brasagem e Fusão - Utilizado para a qualificação de soldadores, operadores de soldagem e procedimentos de soldagem [4].

Segundo Silva Teles [7], a norma ASME B31.3 abrange prescrições sobre projeto, fabricação, montagem, testes e inspeção sobre tubulações para quaisquer fluidos, situadas dentro dos limites das refinarias, terminais e das instalações de processamento, armazenagem e distribuição de produtos de petróleo e de indústrias químicas e petroquímicas em geral.

\subsection{Abordagem ao código ASME B31.3}

A base para devida interpretação do código ASME B31.3 é a coleta de informações do projeto relacionadas ao tipo de tubulação a ser fabricado e/ou montado, mais precisamente ao tipo de fluido que será transportado através destas tubulações. De acordo com o código, esta classificação pode ser definida em: fluido de serviço categoria $D$, fluido de serviço categoria $M$, fluido de serviço de alta pressão e fluido de serviço normal [5].

A compreensão dos tipos de fluidos que serão transportados pela tubulação é uma informação importante para a definição dos parâmetros de soldagem a serem empregados tanto na qualificação do procedimento de soldagem quanto na execução das soldas de fabricação das tubulações. Essa caracterização pode ser melhor compreendida consultando-se o parágrafo 300.2 do ASME B31.3 e com a interpretação do guia de classificação de fluido de serviço, mais especificamente o anexo M do ASME B31.3, ilustrado na Figura 1 [5].

Após a classificação do tipo de fluido de serviço, faz-se necessária a devida interpretação do parágrafo 328.2 no capítulo V, do código ASME B31.3, no qual consta o encaminhamento ao código ASME SECTION IX para que se execute corretamente a qualificação dos procedimentos de soldagem de tubulações industriais [5]. 


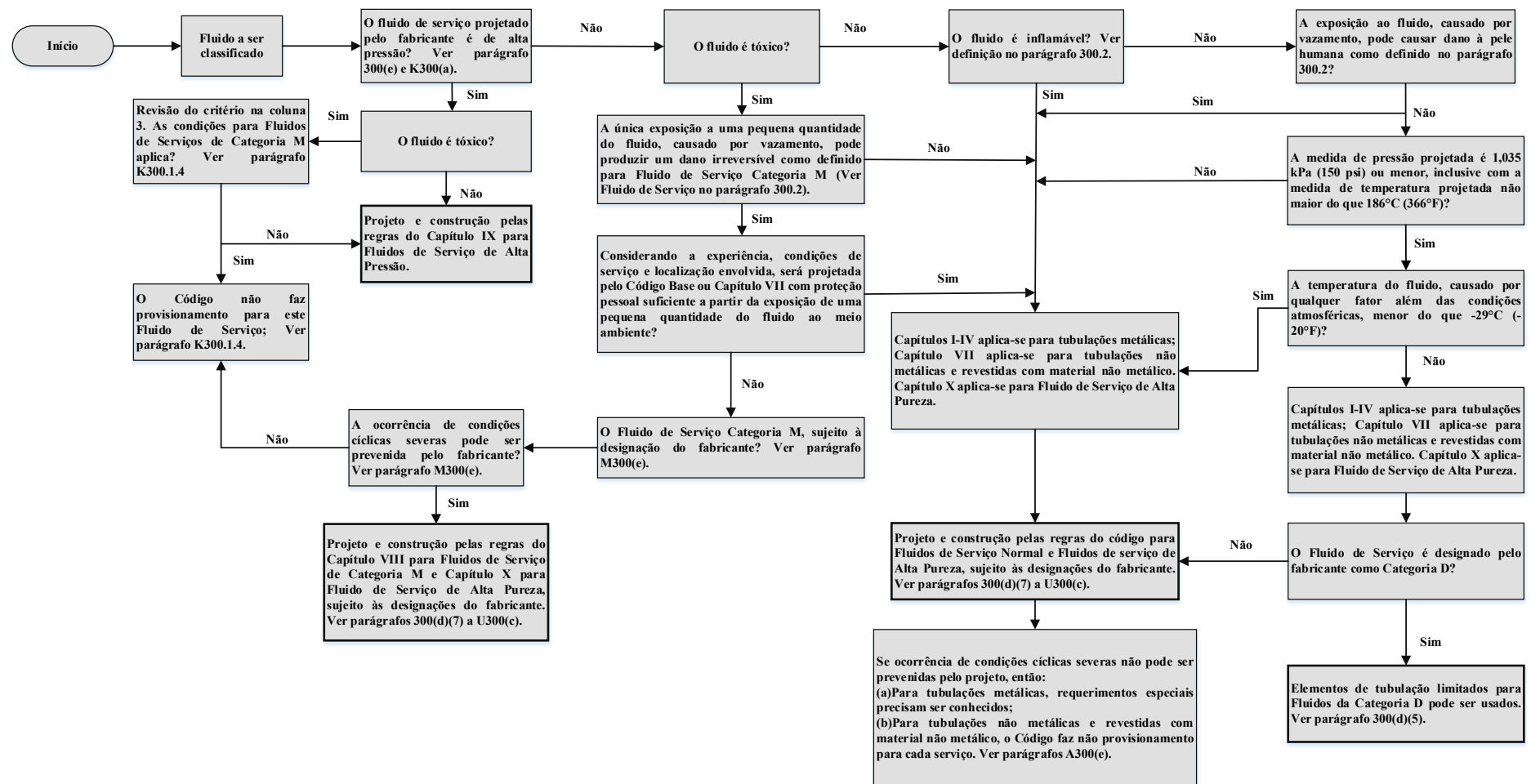

Figura 1. Guia para classificação do fluido de serviço - Fig. M300. Nota: Adaptado de ASME B31.3 [5].

\subsection{Abordagem ao código ASME SECTION IX}

A abordagem deste trabalho será restrita as variáveis essenciais de soldagem por tais informações interferirem diretamente na qualificação ou requalificação do procedimento de soldagem. Neste sentido, analisaremos apenas os parágrafos do código ASME IX cujas características sejam consideradas como variáveis essenciais dos processos de soldagem escolhidos para a fabricação da peça teste: FCAW e GTAW.

Os parágrafos QW-255 e QW-256 do código ASME IX abordam os processos de soldagem FCAW e GTAW [4]. A Tabela 2 reúne as informações das variáveis classificadas como essenciais à qualificação do procedimento de soldagem em ambos processos utilizados na produção da peça teste.

Tabela 2. Variáveis essenciais para qualificação de procedimento de soldagem.

\begin{tabular}{|c|c|c|c|}
\hline \multicolumn{3}{|c|}{ Parágrafos $\times$ Processos de soldagem } & \multirow{2}{*}{ Descrição das variáveis essenciais } \\
\hline Parágrafos & FCAW & GTAW & \\
\hline \multirow{2}{*}{ Metal de base: } & 8 & 8 & Espessura metal de base qualificado \\
\hline & 9 & -- & Espessura do passe $>13 \mathrm{~mm}$ \\
\hline \multirow{2}{*}{ QW-403 } & 10 & -- & Limite de espessura (curto-circuito) \\
\hline & 11 & 11 & P-Number qualificado \\
\hline \multirow{4}{*}{ Metal de adição: } & 4 & 4 & F-Number \\
\hline & 5 & 5 & A-Number \\
\hline & -- & 14 & Adição ou supressão do consumível \\
\hline & 23 & 23 & Formato de consumível \\
\hline \multirow{4}{*}{ QW-404 } & 24 & -- & Consumível suplementar \\
\hline & 27 & -- & Elementos de liga \\
\hline & 30 & 30 & Espessura do metal depositado \\
\hline & 32 & -- & Limite de espessura (curto-circuito) \\
\hline $\begin{array}{c}\text { Pré-aquecimento: } \\
\text { QW-406 }\end{array}$ & 1 & 1 & Decréscimo $>55^{\circ} \mathrm{C}$ \\
\hline Tratamento térmico: & 1 & 1 & Tratamento térmico de alívio de tensões \\
\hline QW-407 & 4 & 4 & Limite de espessura \\
\hline Gás de proteção: & 2 & 2 & Gás simples ou percentual de mistura \\
\hline
\end{tabular}


Tabela 2. Continuação...

\begin{tabular}{|c|c|c|c|}
\hline \multicolumn{3}{|c|}{ Parágrafos $\times$ Processos de soldagem } & \multirow{2}{*}{ Descrição das variáveis essenciais } \\
\hline Parágrafos & FCAW & GTAW & \\
\hline \multirow{2}{*}{ QW-408 } & 9 & 9 & Gás de proteção da raiz \\
\hline & 10 & 10 & Gás de proteção adicional da solda \\
\hline $\begin{array}{l}\text { Características elétricas: } \\
\text { QW-409 }\end{array}$ & 2 & -- & Modo de transferência \\
\hline Técnica: & -- & 11 & Fechamento da câmara orbital \\
\hline QW-410 & 64 & 64 & Uso de processo térmico externo \\
\hline
\end{tabular}

Nota: Adaptado de ASME IX [4].

Todavia, antes de abordarmos as variáveis essenciais, é necessário delimitarmos as dimensões das peças de teste de onde serão retirados os corpos de provas destinados à realização dos ensaios mecânicos $[4,8]$.

\section{Peça Teste para Qualificação do Procedimento de Soldagem}

O formato da peça de teste, se tubo ou chapa, não é considerado uma variável essencial para a qualificação do procedimento de soldagem, pode-se utilizar ambos para tal finalidade, pois conforme QW-211, um procedimento elaborado com chapa permite a execução de soldas em tubos e vice-versa. Uma chapa de teste deve possuir, no mínimo, $250 \mathrm{~mm}$ de comprimento para que seja possível a retirada dos corpos de prova de dobramento e o de tração, este, por sua vez, demostrado no QW-463.1(a) para espécimes com espessura até $19 \mathrm{~mm}$ e QW-463.1 (b) para espécimes acima de $19 \mathrm{~mm}$, conforme ASME IX. A Figura 2 representa o esquema da disposição para retirada dos corpos de prova da chapa de teste conforme ASME IX [4].

\begin{tabular}{|c|c|c|}
\hline Descartar & & Está parte \\
\hline Corpo de prova & & Dobramento lateral \\
\hline Seção reduzida & & Corpo de prova tração \\
\hline Corpo de prova & & Dobramento lateral \\
\hline Corpo de prova & & Dobramento lateral \\
\hline Seção reduzida & & Corpo de prova tração \\
\hline Corpo de prova & & Dobramento lateral \\
\hline Descartar & & Está parte \\
\hline & & \\
\hline
\end{tabular}

(a)

\begin{tabular}{|c|c|c|}
\hline Descartar & & Está parte \\
\hline Seção reduzida & & Corpo de prova tração \\
\hline Corpo de prova & & Dobramento de raiz \\
\hline Corpo de prova & & Dobramento de face \\
\hline Corpo de prova & & Dobramento de raiz \\
\hline Corpo de prova & & Dobramento de face \\
\hline Seção reduzida & & Corpo de prova tração \\
\hline Descartar & & Está parte \\
\hline & & \\
\hline
\end{tabular}

(b)

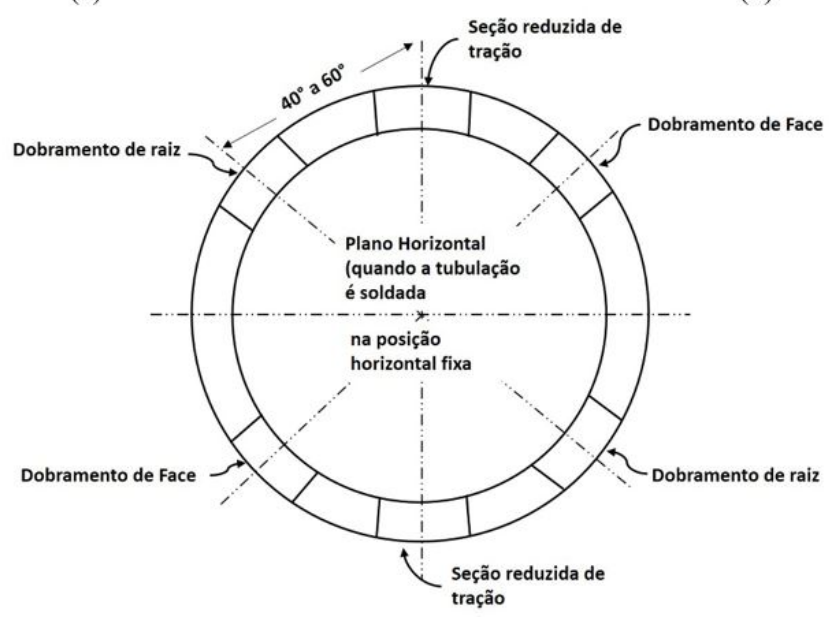

(c)

Figura 2. Detalhe da Figura (a) QW-463.1(a): para chapas menores que $19 \mathrm{~mm}$; (b) QW-463.1(b): para chapas maiores de 19 mm e (c) distribuição de retirada de corpos de prova em tubulação - Adaptado de ASME IX [4]. 
De acordo com as definições de geometria da peça teste, o tipo de chanfro é considerado como variável não essencial. Desta forma, a chapa de teste para qualificação do procedimento de soldagem pode apresentar quaisquer tipos de chanfros: reto, "V", "meio V", “J”, "duplo J”, “U”, "duplo U”, "X" e “K” [4].

\section{Variáveis Essenciais}

\subsection{Metal de base (QW-403)}

O código ASME IX agrupa os metais de base por meio da composição química, soldabilidade e propriedades mecânicas, dessa forma atribui uma denominação chamada de $P$-Number. O item QW-403.11 do ASME IX é o tópico responsável por detalhar os aspectos pertinentes à faixa de qualificação do P-Number, conforme apresentados na Tabela $3[3,4]$.

Tabela 3. Lista de materiais e P-Number - QW/QB-422.

\begin{tabular}{|c|c|c|c|c|c|c|c|}
\hline \multicolumn{8}{|c|}{ Agrupamento de metais para qualificação - QW/QB-422 } \\
\hline \multirow[b]{2}{*}{ Especificação no } & \multirow[b]{2}{*}{ Tipo ou Grau } & \multirow{2}{*}{$\begin{array}{l}\text { Resistência mínima } \\
\text { à tração específica } \\
\text { (MPa) }\end{array}$} & \multirow[b]{2}{*}{ P. № } & \multirow[b]{2}{*}{ Grupo № } & \multirow[b]{2}{*}{$\begin{array}{c}\text { ISO } \\
15608\end{array}$} & \multicolumn{2}{|r|}{ Tipo de Metal Base } \\
\hline & & & & & & $\begin{array}{c}\text { Composição } \\
\text { nominal }\end{array}$ & Forma de produto \\
\hline A/SA-36 & $\ldots$ & $58(400)$ & 1 & 1 & 11.1 & C-Mn-S & Chapa; barra \\
\hline A/SA-53 & Tipo E, Grau A & $48(330)$ & 1 & 1 & 1.1 & $\mathrm{C}$ & Tubos de condução com e sem costura \\
\hline$(\ldots)$ & $(\ldots)$ & $(\ldots)$ & $(\ldots)$ & $(\ldots)$ & $(\ldots)$ & $(\ldots)$ & $(\ldots)$ \\
\hline A/SA-134 & SA2 83 Gr. A & $45(310)$ & 1 & 1 & 1.1 & $\mathrm{C}$ & Tubos soldados \\
\hline A108 & 8620 & $\ldots$ & 3 & 3 & 4.1 & $0,5 \mathrm{Ni}-0,5 \mathrm{Cr}-\mathrm{Mo}$ & Barras \\
\hline$(\ldots)$ & $(\ldots)$ & $(\ldots)$ & $(\ldots)$ & $(\ldots)$ & $(\ldots)$ & $(\ldots)$ & $(\ldots)$ \\
\hline A/SA-204 & $B$ & $70(485)$ & 3 & 2 & 1.1 & $\mathrm{C}-0,5 \mathrm{Mo}$ & Chapa \\
\hline A/AS-209 & $\mathrm{T} 1 \mathrm{~b}$ & $53(365)$ & 3 & 1 & 1.1 & C-0,5Mo & Tubos sem costura \\
\hline$(\ldots)$ & $(\ldots)$ & $(\ldots)$ & $(\ldots)$ & $(\ldots)$ & $(\ldots)$ & $(\ldots)$ & $(\ldots)$ \\
\hline A/AS-249 & TP XM-19 & $100(690)$ & 8 & 3 & 8.3 & $22 \mathrm{Cr}-13 \mathrm{Ni}-5 \mathrm{Mn}$ & Tubos soldados \\
\hline
\end{tabular}

Nota: Adaptado de ASME IX [4].

Os detalhes relativos à faixa de espessura de qualificação do metal de base e número de corpos de prova para testes podem ser encontrados no QW-403.8 e na tabela QW-451.1, a qual podem ser observados na Tabela 4 [4].

Tabela 4. Faixa de espessura e testes mecânicos para qualificação de procedimento - QW-451.1.

\begin{tabular}{|c|c|c|c|c|c|c|c|}
\hline \multicolumn{8}{|c|}{ Teste de Tração e de Dobramento Transversal } \\
\hline \multirow{2}{*}{$\begin{array}{l}\text { Espessura T do metal de base } \\
\text { soldado para testes ( } \mathrm{mm})\end{array}$} & \multicolumn{2}{|c|}{$\begin{array}{c}\text { Faixa de espessura } \\
\text { de metal base } \\
\text { qualificada }(\mathrm{mm})\end{array}$} & \multirow{2}{*}{$\begin{array}{l}\text { Espessuras do metal de } \\
\text { solda qualificadas em } \\
\text { função da espessura t do } \\
\text { metal de solda } \\
\text { depositado (mm) }\end{array}$} & \multicolumn{4}{|c|}{$\begin{array}{l}\text { Tipo e quantidade dos testes requeridos [Teste de tração } \\
\text { e dobramento guiado] }\end{array}$} \\
\hline & Mín. & Máx. & & $\begin{array}{l}\text { Tração [QW- } \\
\text { 150] }\end{array}$ & $\begin{array}{l}\text { Dobramento } \\
\text { lateral [QW- } \\
\quad 160]\end{array}$ & $\begin{array}{l}\text { Dobramento } \\
\text { de face [QW- } \\
\text { 160] }\end{array}$ & $\begin{array}{l}\text { Dobramento } \\
\text { de raiz [QW- } \\
\quad 160]\end{array}$ \\
\hline Menor do que 1,5 & $\mathrm{~T}$ & $2 \mathrm{~T}$ & $2 t$ & 2 & {$[\ldots]$} & 2 & 2 \\
\hline Entre 1,5 a 10 & 1,5 & $2 \mathrm{~T}$ & $2 \mathrm{t}$ & 2 & {$[\ldots]^{5}$} & 2 & 2 \\
\hline Acima de 10 e menor do que 19 & 5 & $2 \mathrm{~T}$ & $2 t$ & 2 & {$[\ldots]^{5}$} & 2 & 2 \\
\hline Entre 19 a 38 & 5 & $2 \mathrm{~T}$ & $2 t$ quando $t<19$ & 2 & 4 & {$[\ldots]$} & {$[\ldots]$} \\
\hline Entre 19 a 38 & 5 & $2 \mathrm{~T}$ & $2 T$ quando $t \geq 19$ & 2 & 4 & {$[\ldots]$} & {$[\ldots]$} \\
\hline Entre 38 a 150 & 5 & 200 & $2 \mathrm{t}$ quando $\mathrm{t}<19$ & 2 & 4 & {$[\ldots]$} & {$[\ldots]$} \\
\hline Entre 38 a 150 & 5 & 200 & 200 quando $t \geq 19$ & 2 & 4 & {$[\ldots]$} & {$[\ldots]$} \\
\hline Maior do que 150 & 5 & $1,33 \mathrm{~T}$ & $2 t$ quando $t<19$ & 2 & 4 & {$[\ldots]$} & {$[\ldots]$} \\
\hline Maior do que 150 & 5 & $1,33 \mathrm{~T}$ & $1,33 T$ quando $t \geq 19$ & 2 & 4 & {$[\ldots]$} & {$[\ldots]$} \\
\hline
\end{tabular}

${ }^{5}$ Os dobramentos de face e raiz podem ser substituidos por quatro dobramentos laterais para espessuras $\geq 10 \mathrm{~mm}$. Fonte: Adaptado de ASME IX [4].

O código ASME IX tem inúmeras notas e exceções, dentre estas as QW403.9 e QW-403.10, que especificam quando se utiliza o processo de soldagem GMAW: se uma peça de teste for soldada com este processo, sejam os passes simples ou múltiplos com espessura maior que $13,0 \mathrm{~mm}$, a faixa de qualificação do procedimento será de até 1,1 vezes a faixa aplicada na peça de teste. 


\subsection{Consumível de soldagem (QW-404)}

Os consumíveis de soldagem são agrupados de acordo com usabilidade e características, que de acordo com a norma influem na habilidade dos soldadores e operadores quanto a execução da solda. A nomenclatura de composição é denominada F-Number e A-Number, sendo a mais importante delas o F-Number devido ao A-Number ser restrito apenas aos materiais ferrosos. A Tabela 5 exemplifica a classificação dos consumíveis de soldagens conforme QW-432 do código ASME IX [4].

Tabela 5. Faixa de F-Number - QW-432.

\begin{tabular}{|c|c|c|c|}
\hline F. № & Especificação № & Classificação AWS & UNS № \\
\hline \multicolumn{4}{|c|}{ Aço carbono e aço liga } \\
\hline 1 & SFA-5 .1 & EXX2 0 & $\ldots$ \\
\hline 1 & SFA-5 .1 & EXX2 2 & $\ldots$ \\
\hline 2 & SFA-5.1 & EXX12 & $\ldots$ \\
\hline 2 & SFA-5 .1 & EXX13 & $\ldots$ \\
\hline 3 & SFA-5 .1 & EXX10 & $\ldots$ \\
\hline$(\ldots)$ & $(\ldots)$ & $(\ldots)$ & $(\ldots)$ \\
\hline
\end{tabular}

Nota: Adaptado de ASME IX [4].

Diversas outras variáveis essenciais relativas aos consumíveis de soldagem são controladas pela norma citada: adição ou supressão do consumível (QW-404.14), forma de produção do consumível (QW-404.23), consumível suplementar (QW-404.24), elementos de liga (QW-404.27), espessura do metal depositado (QW-404.30).

\subsection{Condições de pré-aquecimento (QW-406) e TTAT (QW-407)}

Inicialmente, o QW-406.1 apresenta as condições de pré-aquecimento e os QW-407.1 e QW-407.4 detalham as características relativas à realização do tratamento térmico de alívio de tensões (TTAT), de acordo com o ASME IX. Estas variáveis são melhores detalhadas nas tabelas 330.1.1 e 331.1.1 do código ASME B31.3. Tem-se, na Tabela 6, exemplos das faixas de aplicação do pré-aquecimento contidas no B31.3 [5].

Tabela 6. Faixa de aplicação de pré-aquecimento - tabela 330.1.1.

\begin{tabular}{|c|c|c|c|c|}
\hline $\begin{array}{c}\text { P-Number metal } \\
\text { de base }\end{array}$ & Grupo metal de base & Espessura (mm) & Limites adicionais & $\begin{array}{c}\text { Temperatura mínima } \\
\text { requerida }\left({ }^{\circ} \mathrm{C}\right)\end{array}$ \\
\hline & & $\leq 25$ & - & 10 \\
\hline \multirow[t]{3}{*}{1} & Aço carbono & $>25$ & $\% C \leq 0,03$ & 10 \\
\hline & & $>25$ & $\% C>0,03$ & 95 \\
\hline & & $\leq 13$ & SMTS $\leq 450 \mathrm{Mpa}$ & 10 \\
\hline \multirow[t]{2}{*}{2} & Ligas de aço; $\mathrm{Cr} \leq 0,5 \%$ & $>13$ & SMTS $\leq 450 \mathrm{Mpa}$ & 95 \\
\hline & & Todas & SMTS > $450 \mathrm{Mpa}$ & 95 \\
\hline 3 & Ligas de aço; $0,5<\mathrm{Cr} \leq 2,0 \%$ & Todas & {$[\ldots]$} & 120 \\
\hline
\end{tabular}

Nota: Adaptado de ASME B31.3 [5].

Assim como o pré-aquecimento, o tratamento térmico pós soldagem é um item considerado variável essencial. O código ASME B31.3 especifica claramente que a necessidade de pré-aquecimento e a tratamento térmico a serem utilizados devem ser especificados no projeto de engenharia e demonstrado pela qualificação do procedimento. A Tabela 7 apresenta um recorte das especificações deste mesmo código para o TTAT.

Tabela 7. Especificação de requisitos para tratamento térmico - tabela 331.1.1.

\begin{tabular}{|c|c|c|c|c|c|c|c|}
\hline $\begin{array}{l}\text { P-Number } \\
\text { metal de } \\
\text { base }\end{array}$ & A - Number & Grupo metal de base & $\begin{array}{c}\text { Espessura } \\
\text { (mm) }\end{array}$ & $\begin{array}{c}\text { Resistência } \\
\text { mínima a tração } \\
\text { (MPa) }\end{array}$ & $\begin{array}{c}\text { Faixa de } \\
\text { temperatura }\left({ }^{\circ} \mathrm{C}\right)\end{array}$ & $\begin{array}{l}\text { Tempo de } \\
\text { espera } \\
(\mathrm{min} / \mathrm{mm})\end{array}$ & $\begin{array}{c}\text { Tempo } \\
\text { mínimo (h) }\end{array}$ \\
\hline \multirow{2}{*}{1} & \multirow{2}{*}{1} & \multirow{2}{*}{ Aço carbono } & $\leq 19$ & Todas & - & - & - \\
\hline & & & $>19$ & Todas & $593-649$ & 2,4 & 1 \\
\hline \multirow{2}{*}{3} & \multirow{2}{*}{$2 ; 11$} & \multirow{2}{*}{ Aços liga ( $\mathrm{Cr} \leq 0,5 \%)$} & $\leq 19$ & $\leq 490$ & - & - & - \\
\hline & & & $>19$ & Todas & $593-718$ & 2,4 & 1 \\
\hline \multirow{2}{*}{6} & \multirow{2}{*}{6} & Aços alta liga & Todas & Todas & $732-788$ & 2,4 & 2 \\
\hline & & A 240 Gr. 429 & Todas & Todas & $621-663$ & 2,4 & 2 \\
\hline
\end{tabular}

Nota: Adaptado de ASME B31.3 [5]. 
De acordo com as condições aplicadas no estudo de caso, P-number 1 (aço carbono) com espessura de 12,5 mm, conforme Tabela 7, não é aplicável pré-aquecimento nem tratamento térmico.

\subsection{Gás (QW-408), características elétricas (QW-409) e técnica de soldagem (QW-410)}

De acordo com o parágrafo QW-408.2, faz-se necessário a requalificação do procedimento de soldagem em caso de alteração do tipo de gás, se simples ou misturado. Considerando-se o parágrafo QW-408.9, um procedimento qualificado com gás de proteção na raiz, isto é, gás de purga, deve ser empregado apenas com gás de purga. Por outro lado, um procedimento qualificado sem gás de purga pode ser empregado para soldagens com ou sem gás de proteção na raiz [4].

Em relação às características elétricas, para o processo FCAW, faz-se necessária a requalificação do procedimento de soldagem caso ocorra uma mudança no modo de transferência metálica, alteração do modo globular, spray ou spray pulsado para o modo de transferência por curto-circuito, ou vice-versa [4].

O parágrafo QW-408.10, juntamente com os requisitos inerentes à técnica de soldagem, mais especificamente os parágrafos QW-410.11 e QW-410.64, são pouco aplicáveis ao tema em estudo [4].

\section{Aplicação da Metodologia}

\subsection{Desenvolvimento dos parâmetros de soldagem}

Considerando as variáveis normativas apresentadas, como peça de teste para o estudo de caso foi utilizado uma chapa do aço ASTM A-516 grau 70, classificação P-Number 1 e nas dimensões de $400 \mathrm{~mm}$ de comprimento por $200 \mathrm{~mm}$ de largura com $12,7 \mathrm{~mm}$ de espessura. O chanfro foi em " $V$ " e a posição de soldagem adotada foi a vertical (3G) na progressão ascendente. Na Figura 3 é apresentada a chapa teste para estudo de caso apresentado nesse trabalho. 

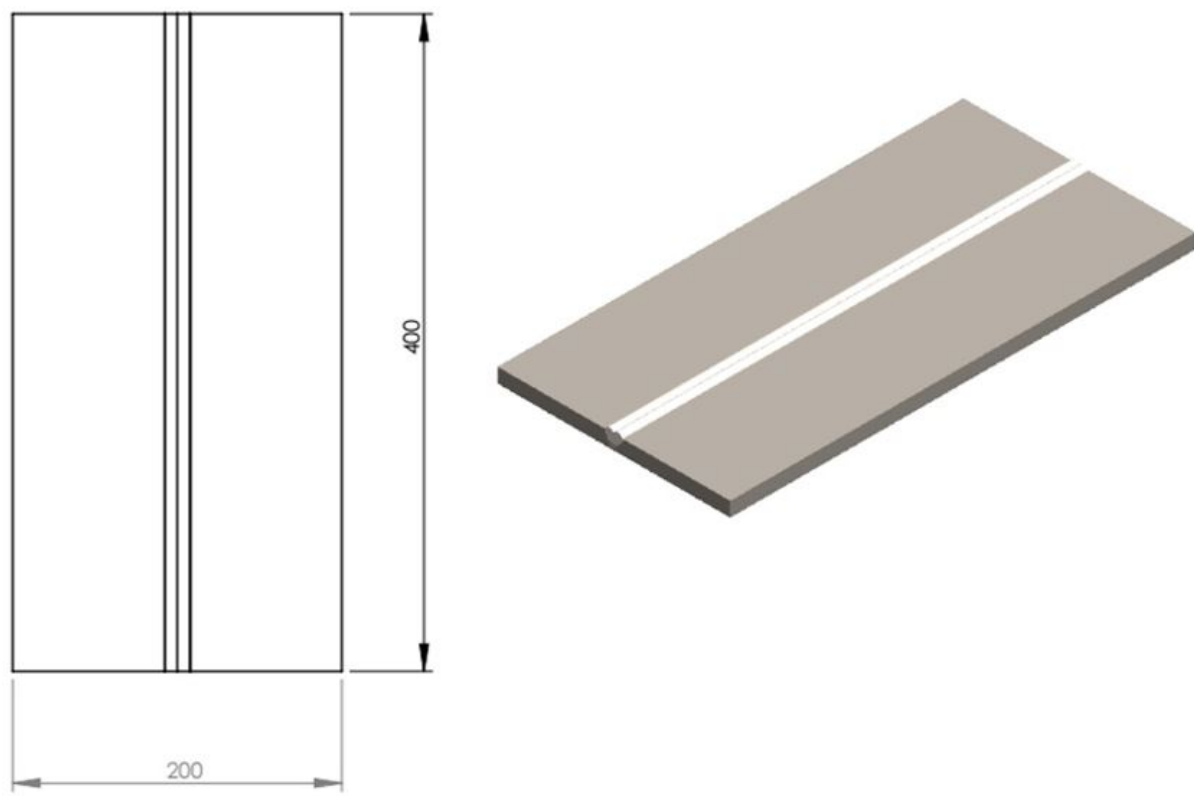

(a)
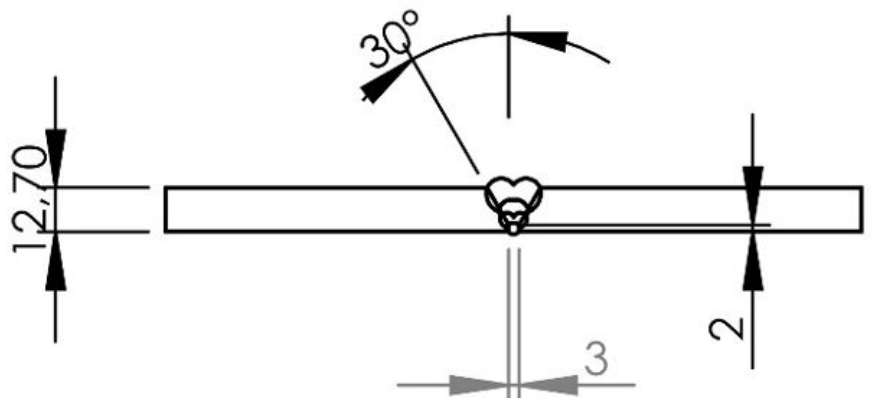

(b)

Figura 3. Croqui da chapa de teste para produção dos corpos de prova (a) detalhes das dimensões da chapa teste (b) detalhes da junta.

A soldagem foi realizada por um soldador qualificado. Foi utilizado o processo GTAW na raiz e o FCAW no enchimento e acabamento da junta, amplamente utilizados para a soldagem de tubulações industriais [9]. Os parâmetros de soldagem foram controlados e registrados no RQPS, por conseguinte, foi possível a emissão da EPS. Para o processo de soldagem TIG, a faixa de corrente foi de 135 a 155 Ampères [9] e para o processo de soldagem por arame tubular a faixa de corrente foi de 140 a 145 Ampères [1].

Os consumíveis utilizados foram de classificação ER-70S-3, para o GTAW - com o emprego do gás argônio - e arame tubular E-71T-1, para o FCAW - com o gás de proteção $\mathrm{CO}_{2}$. A temperatura de interpasse foi controlada, por meio de lápis térmico, limitando a temperatura até 250 ㄷ. Segundo o ASME B31.3, para o material empregado nesta atividade, não há necessidade de aplicação de pré-aquecimento e tratamento térmico de alívio de tensões [5].

\subsection{Realização dos Ensaios Não Destrutivos (END)}

Após a realização das soldagens, o ensaio visual foi efetuado para a verificação de possíveis descontinuidades ou defeitos característicos da soldagem de acordo com o parágrafo QW-194. Adicionalmente, um ensaio por líquido penetrante foi executado para a averiguação de existência de descontinuidades não detectadas pelo ensaio visual, conforme mostra a Figura 4. Os resultados dos ensaios foram considerados aprovados de acordo com os critérios da ASME IX [4]. 


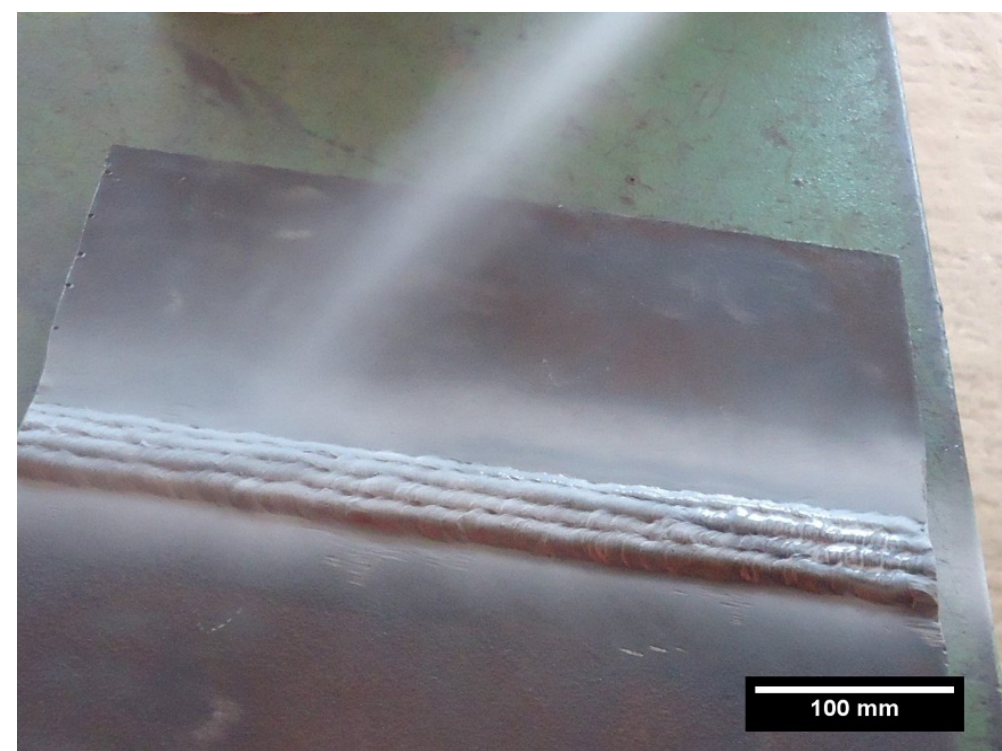

Figura 4. Realização do ensaio por líquido penetrante.

\subsection{Realização dos Ensaios Mecânicos}

Conforme ASME IX [4], para o ensaio de dobramento, foram retirados quatro corpos de prova conforme esquema apresentado na Figura 2a, os quais foram identificados como DL-1, DL-2, DL-3 e DL-4. A Figura 5a, 5b e 5c, a seguir, apresentam a sequência de execução do ensaio de dobramento e a Figura 5d demonstra o corpo de prova já dobrado.

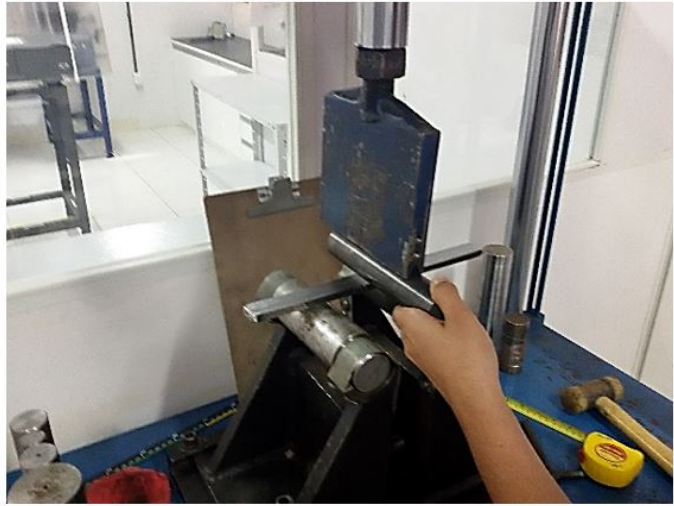

(a)

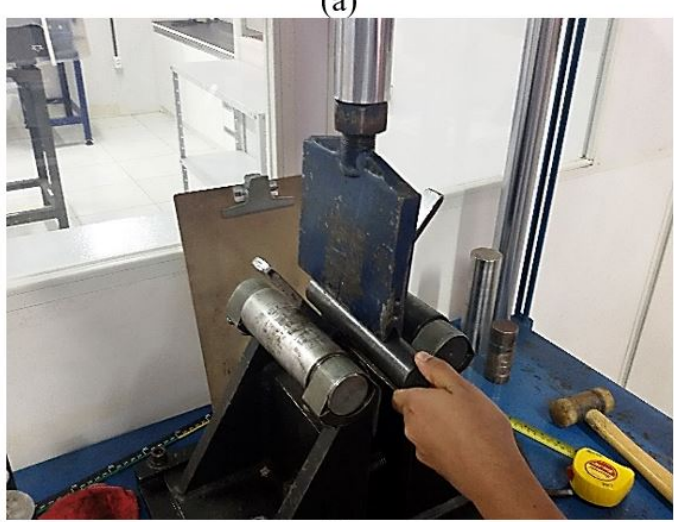

(c)

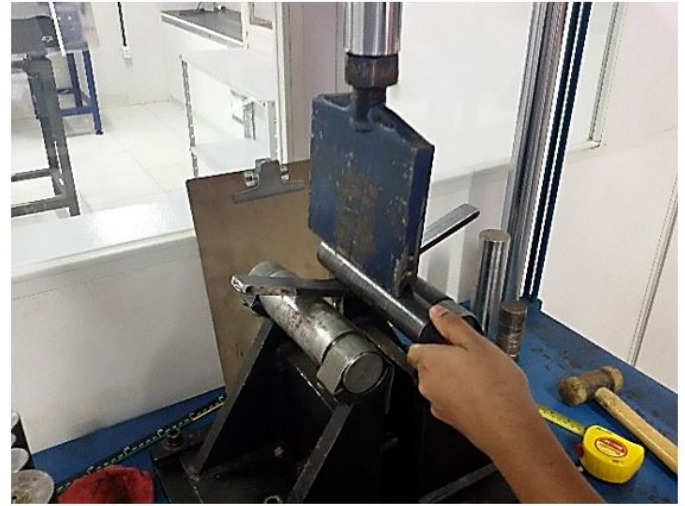

(b)

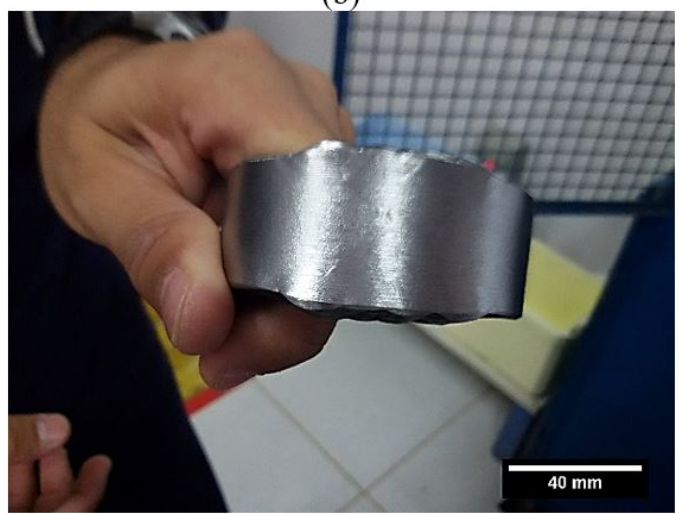

(d)

Figura 5. Registro do ensaio de dobramento lateral: ( $a, b$ e c) sequência de execução do ensaio de dobramento e (d) corpo de prova após dobrado. 
Os resultados foram analisados de acordo com parágrafo QW-163, não sendo identificadas descontinuidades na região da solda ou no metal de base, validando os corpos de prova no teste conforme critérios da ASME IX [4]. A Tabela 8 apresenta os resultados registrados no relatório de ensaio mecânico de dobramento, os quais foram considerados satisfatórios.

Tabela 8. Resultados dos ensaios de dobramento.

\begin{tabular}{cll}
\hline Corpo de prova & Dimensões $(\mathbf{m m})$ & \multicolumn{1}{c}{ Resultados } \\
DL-1 & $10,0 \times 12,7 \times 200$ & Isentos de descontinuidades \\
DL-2 & $10,0 \times 12,7 \times 200$ & Isentos de descontinuidades \\
DL-3 & $10,0 \times 12,7 \times 200$ & Isentos de descontinuidades \\
DL-4 & $10,0 \times 12,7 \times 200$ & Isentos de descontinuidades \\
\hline
\end{tabular}

Nota: preparação do corpo de prova (QW-462.2), método de ensaio (QW-162) e critério de aceitação (QW-163), conforme ASME IX [4].

Para o ensaio de tração, de acordo com o ASME IX [4], foram preparados dois corpos de prova, identificados como T-1 e T-2. A Figura 6 mostra a evolução do ensaio: (a) início do ensaio, (b) o exato momento da ruptura do corpo-de-prova na imagem, em (c) esquema representando o local de fratura do corpo de prova e as imagens (d) e (e) apresentam os corpos de prova já ensaiados, mostrando a corpo de prova fraturado no metal de base e a superfície de fratura com aspecto taça-cone, respectivamente. Essa característica é típica de materiais com apreciável deformação plástica antes da fratura [10]. 


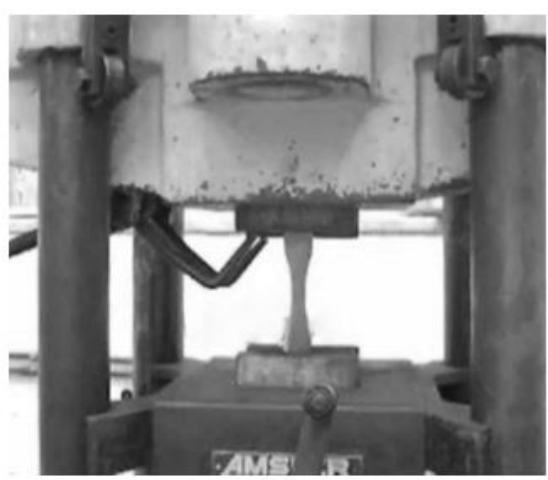

(a)

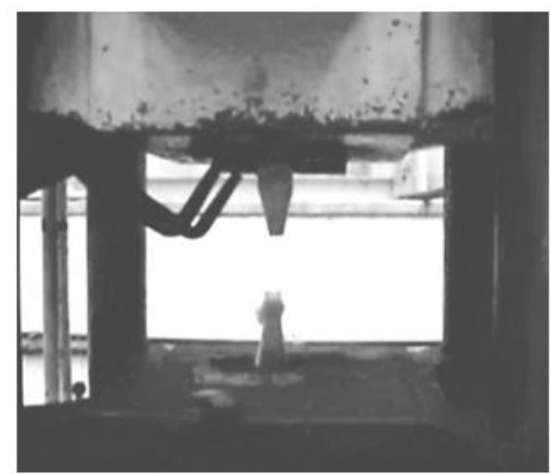

(b)
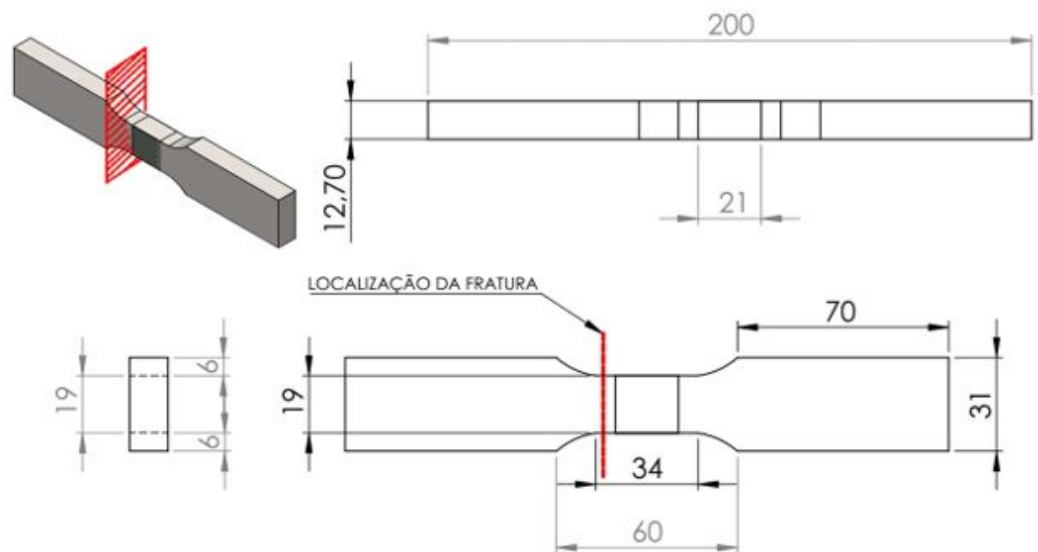

(c)

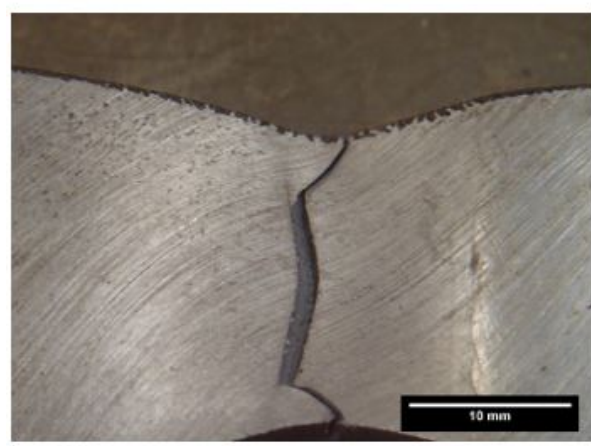

(d)

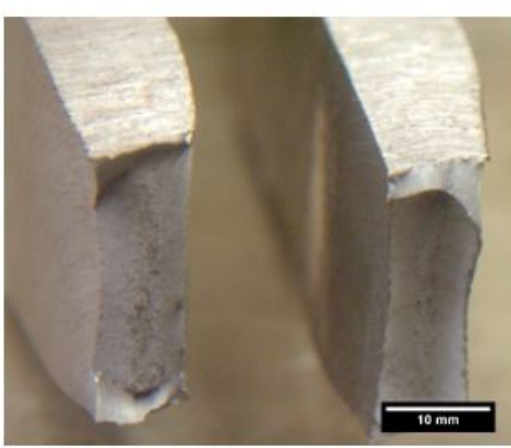

(e)

Figura 6. Sequência de execução do ensaio de tração: (a) início do ensaio; (b) momento da ruptura.; (c) esquema com indicação do local da fratura e (d) e (e) corpos de prova após ensaio.

Os resultados, apresentados na Tabela 9, foram analisados de acordo com o critério de aceitação do ensaio de resistência à tração contidos no parágrafo QW-153 do ASME IX e foram considerados aprovados [4].

Tabela 9. Resultado do ensaio mecânico de resistência à tração.

\begin{tabular}{|c|c|c|c|c|c|c|c|}
\hline Corpo de prova & Largura (mm) & Espessura (mm) & Área $\left(\mathrm{mm}^{2}\right)$ & $\begin{array}{l}\text { Carga de } \\
\text { ruptura } \\
\text { (Kgf) }\end{array}$ & $\begin{array}{c}\text { Tensão de } \\
\text { ruptura (MPa) }\end{array}$ & Local de ruptura & Laudo \\
\hline $\mathrm{T}-1$ & 19,2 & 12,1 & 232,3 & 11.250 & 485,0 & Metal de base & Aprovado \\
\hline $\mathrm{T}-2$ & 19,3 & 12,4 & 239,3 & 11.225 & 483,0 & Metal de base & Aprovado \\
\hline
\end{tabular}

Nota: preparação do corpo de prova (QW-462.1(A)), método de ensaio (QW-152) e critério de aceitação (QW-153), conforme ASME IX [4].

A aplicação de ensaio de impacto é prevista pela norma ASME B31.3 [5] para as condições especificadas no parágrafo 323.2.2; tabela 323.2.2A e complementadas pelas figuras 323.2.2A e 323.2.2B; projeto de engenharia ou quando requerido por seções adicionais do código ASME. A Seção IX da ASME recomenda, conforme QW-172, seguir os critérios de aceitação de acordo 
com a norma base do projeto, neste caso ASME B31.3[4]. De acordo com os critérios estabelecidos (nota 5 da tabela 323.2.2A), não houve necessidade de aplicação de ensaio de impacto para as condições do estudo de caso, pois a condição operacional para o estudo em questão prever operação em temperatura ambiente.

\subsection{Elaboração do RQPS e EPS}

Com os resultados e os relatórios dos ensaios mecânicos, foi elaborado o Registro de Qualificação do Procedimento de Soldagem (RQPS). Considerando as informações contidas no parágrafo QW-200.1, na qual afirma que todo procedimento de soldagem deve estar suportado por um RQPS, a partir destas informações foi possível elaborar a EPS, o qual seu conteúdo é ilustrado na Figura 7, sendo este o procedimento para soldagem de tubulações industriais desenvolvido neste trabalho.

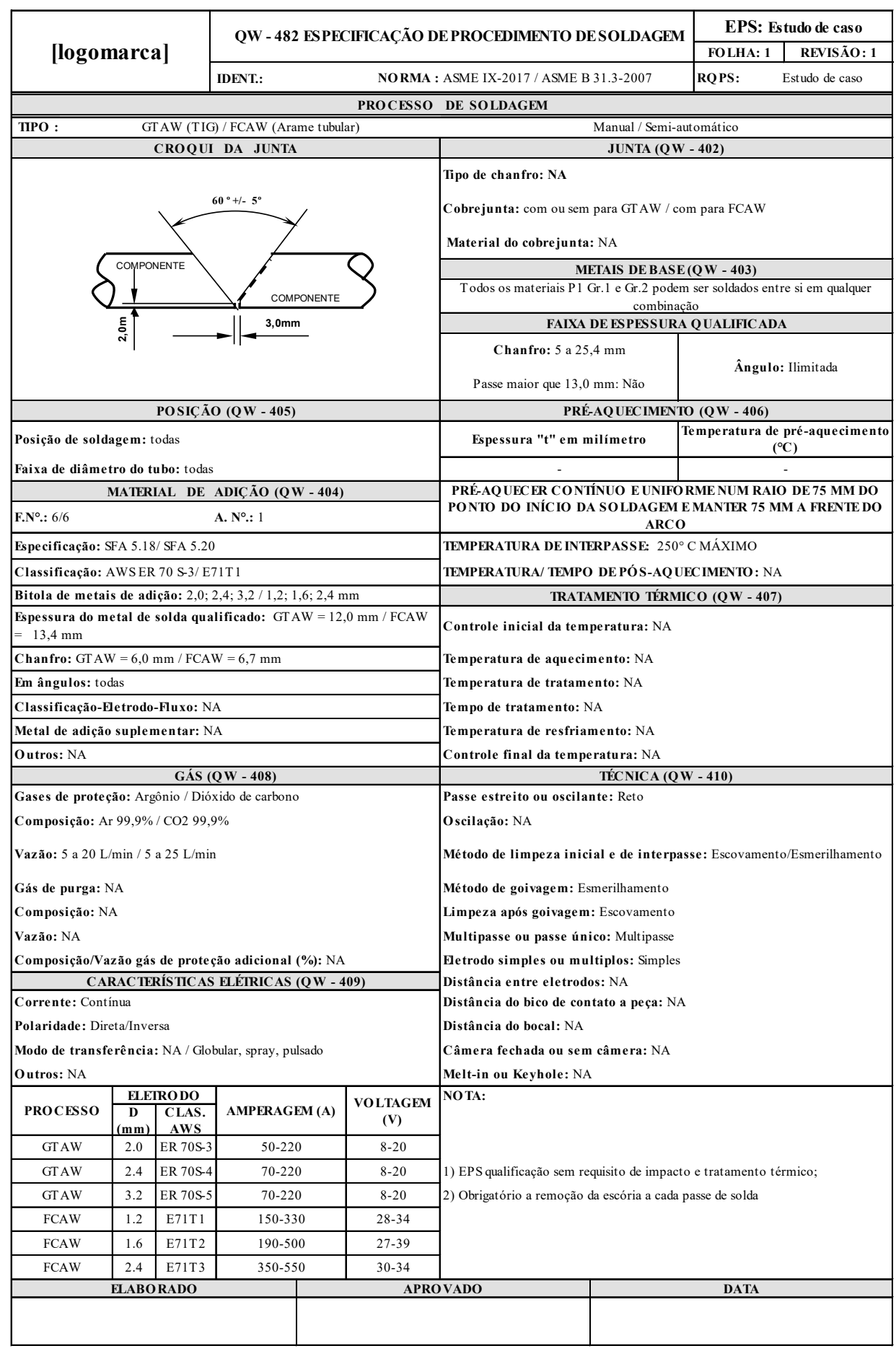

Figura 7. Especificação do Procedimento de Soldagem (EPS). 


\section{Conclusão}

Este trabalho discutiu a metodologia de qualificação de procedimentos de soldagem de forma simples e objetiva, assim como a forma correta de condução dos testes e ensaios mecânicos e a consequente análise dos resultados.

Com base no conhecimento dos critérios estabelecidos pelas normas referenciadas e por meio dos resultados dos ensaios mecânicos, os quais foram classificados como aprovados, foi possível a elaboração do Registro de Qualificação e Procedimento de Soldagem (RQPS) e da Especificação de Procedimento de Soldagem (EPS), a qual pode ser aplicada à soldagem de fabricação e montagem de tubulações industriais de processo, bem como aos serviços de reparo destes equipamentos.

\section{Agradecimentos}

Os autores agradecem à FACEPE, CNPq, CAPES, ANP/FINEP, à empresa WPS Equipamentos pela doação do material de estudo e ao suporte técnico do inspetor de soldagem Vagner Macena.

\section{Referências}

[1] Marques PV, Modenesi PJ, Bracarense AQ. Soldagem - fundamentos e tecnologia. Belo Horizonte: Editora UFMG; 2005.

[2] Silva RM. Avaliação de tensões residuais e validação da resistência mecânica de juntas soldadas conforme ASME IX 2017 [dissertação de mestrado]. Uberlândia: Universidade Federal de Uberlândia, Faculdade de Engenharia Mecânica; 2018.

[3] Wang Z, Yang Y. Comparison of welder performance qualification rules between Chinese regulation and ASME BPVS Sec. IX-2015. In: American Society of Mechanical Engineers. Proceedings of the ASME 2016: Pressure Vessels and Piping Conference; 2016 July 17-21; Vancouver, Canada. New York: ASME; 2016. p. V01BT01A025. https://doi.org/10.1115/PVP2016-63194.

[4] American Society of Mechanical Engineers. ASME section IX: qualification standard for welding and brazing procedures, welders, brazers, and welding and brazing operators. IX ed. New York: ASME; 2017.

[5] American Society of Mechanical Engineers. ASME B31.3: process piping. New York: ASME; 2014.

[6] Fundação Brasileira de Tecnologia da Soldagem. Certificação da qualidade [página da internet]. Rio de Janeiro: FBTS; 2018 [acesso em 05 jun. 2018]. Disponível em: http://www.fbts.org.br/

[7] Teles PCS. Tubulações industriais: materiais, projeto, montagem. 10. ed. São Paulo: Cromossete, 2008.

[8] Askeland DR, Phulé PP. Ciência e engenharia dos materiais. São Paulo: Cengage Learning, 2008. 594 p.

[9] Figueiredo KM. Tecnologia da soldagem. São Luís: UFRN; 2005.

[10] American Society for Metals. Handbook ASM. Vol. 12, Fractography. Ohio: ASM International; 1987. 517 p. 